\title{
ON CONVERGENCE FACTORS IN DOUBLE SERIES AND THE
}

\section{DOUBLE FOURIER'S SERIES*}

\author{
BY \\ CHARLES N. MOORE
}

Introduction.

It is generally recognized that in order to show that an actual solution of the physical problem is given by the formal series arising in many problems of mathematical physics, it is necessary to apply the theory of convergence factors to these series. Results in this direction of the greatest generality have been obtained by a method originated by FEJÉr, $\dagger$ and applied by him to problems involving the ordinary Fourier's series.

In a previous paper $\ddagger$ the writer bas applied FEJÉR's method to problems that involve developments in Bessel's functions. The object of the present paper is to make such an application to problems that involve the development of a function of two variables in a double Fourier's series. Such a discussion naturally involves the consideration of certain general facts in the theory of the summability of double series, a theory that has as yet been scarcely touched on. We have not made, however, any attempt to found a very broad theory of this sort. Such an attempt would have led us too far from the central aim of the paper, and in any case it seems preferable that the theory of summability of double series should be developed at first from the point of view of its applications, and that the study of such a theory from the abstract point of view can be made to better advantage when the facts of widest use in the application of the theory have been brought to light.

The consideration of the summability of double series naturally suggests the consideration of the summability of multiple series of any order, and the study of the summability of the double Fourier's series suggests the study of the summability of the triple Fourier's series and the problems connected with it. Generalizations of the results of the present paper to such cases may be obtained by methods similar to those employed in the subsequent discussion.

* Presented to the Society, April 29, 1911, and December 27, 1911.

$\dagger$ Cf. his well-known memoir, Untersuchungen über Fouriersche Reihen, M a th e mat i s ch e Annalen, vol. 58 (1903-04), p. 51 .

$\ddagger$ These Transactions, vol. 10 (1909), p. 391. 
$\$ 1$. Summability of Double Series. Definitions and General Facts.

We will begin this section by defining the type of summability of double series that we shall be concerned with in this paper. Consider the double series

$$
\begin{array}{r}
a_{11}+a_{12}+a_{13}+\cdots \\
+a_{21}+a_{22}+a_{23}+\cdots \\
+a_{31}+a_{32}+a_{33}+\cdots
\end{array}
$$

and form

where

$$
S_{m n}^{(k)}=\sum_{i=1, j=1}^{m, n} \frac{\Gamma(k+m-i)}{\Gamma(k) \Gamma(m-i+1)} \cdot \frac{\Gamma(k+n-j)}{\Gamma(k) \Gamma(n-j+1)} s_{i j},
$$

If the quotient

$$
s_{i j}=\sum_{p=1, q=1}^{i, j} a_{p q} .
$$

where

$$
\frac{S_{m n}^{(k)}}{A_{m n}^{(k)}}
$$

$$
A_{m n}^{(k)}=\frac{\Gamma(m+k)}{\Gamma(k+1) \Gamma(m)} \cdot \frac{\Gamma(n+k)}{\Gamma(k+1) \Gamma(n)},
$$

approaches a limit as $m$ and $n$ become infinite, the double series (1) will be said to be summable $(C k)^{*}$ and to have a value equal to this limit. This type of summability for double series is seen to be entirely analogous to the type of summability for simple series first considered by Cesìro.

In the above definition $k$ may be any quantity real or complex, except zero or a negative integer. The definition may at once be extended to the case $k=0$, if we assume that for this value of $k$ the right-hand sides of (2) and (5) have the value that they approach as $k$ approaches zero. It is then seen that summability $(C O)$ is the same as convergence. $\dagger$ In this paper we shall restrict ourselves to the cases where $k$ is a positive integer or zero. The discussion of non-integral orders of summability for double series seems to have no special bearing on the applications we wish to make, and would therefore lead us too far away from the central object of this investigation.

We now wish to develop some properties of $S_{m n}^{(k)}$ and $A_{m n}^{(k) !}$. It is evident

* We follow here a notation introduced by G. H. Hardr. See Proceedings of the London Mathematical Society, ser. 2, vol. 6 (1908), p. 257.

† We refer here to convergence as defined by Prinashorm. See M ü n chener S i tzu n g b e r i ch t e, vol. 27 (1897), p. 101, and Encyklopddie der mathematischen Wissenschaften, vol. I, A 3, p. 97. Also cf. Bromwick, Theory of Infinite Series, Chap. V.

¥ Non-integral orders of summability of ordinary series have been discussed recently by Chapman, Knopp, Otrounnam, and M. Rress. 
from the definitions of these quantities that

$$
\begin{gathered}
\sum_{m=1, n=1}^{\infty} S_{m n}^{\infty} S^{(k)} y^{n} \sim(1-x)^{-k}(1-y)^{-k} \sum_{m=1, n=1}^{\infty} s_{m n}^{\infty} x^{m} y^{n}, \\
\sum_{m=1, n=1}^{\infty, \infty} A_{m n}^{(k)} x^{m} y^{n} \sim x y(1-x)^{-(k+1)}(1-y)^{-(k+1)},
\end{gathered}
$$

where the sign $\sim$ is used in the sense that if the expressions on the two sides of this sign are expanded in ascending powers of $x$ and $y$. the coefficients of corresponding terms on the two sides will be equal. The second expression could obviously be written as an equality, since the series on both sides converge absolutely when $x$ and $y$ are less in absolute value than unity. Furthermore, it would not be difficult to show that the same is true of the first relation for the type of series considered in this paper. However, as the expansions are used merely as a device to obtain more simply certain relations between the $S$ 's, the $s$ 's, the $a$ 's, and the $A$ 's, relations which depend only on the equations (2), (3), and (5), and which subsist whether the expansions in (6) are convergent or not, it does not seem worth while to consider the question of convergence.

We have at once from (7)

$\sum_{1,1}^{\infty} A_{m n}^{(k)} x^{m} y^{n} \sim x y\left(1+x+x^{2}+\cdots\right)\left(1+y+y^{2}+\cdots\right)(1-x)^{-k}(1-y)^{-k}$, and on equating the coefficients of $x^{m} y^{n}$ on the two sides, we obtain

$$
A_{m n}^{(k)}=\sum_{k=1, j=1}^{m, n}\left(\begin{array}{c}
k+m-i-1 \\
k-1
\end{array}\right)\left(\begin{array}{c}
k+n-j-1 \\
k-1
\end{array}\right),
$$

so that the denominator in the quotient (4) is equal to the sum of the coefficients of the $s$ 's in the numerator. That is to say, we are dealing here with a weighted mean of the sums, just as in Cesìno's method for ordinary series.

Furthermore, since obviously

$$
\sum_{1,1}^{\infty, \infty} s_{m n} x^{m} y^{n} \sim\left(1+x+x^{2}+\cdots\right)\left(1+y+y^{2}+\cdots\right) \sum_{1,1}^{\infty \infty} a_{m n} x^{m} y^{n},
$$

it follows from (6) that

$$
(1-x)^{k+1}(1-y)^{k+1} \sum_{1,1}^{\infty} S_{m n}^{(k)} x^{m} y^{n} \sim \sum_{1,1}^{\infty} a_{m n} x^{m} y^{n},
$$

whence, equating coefficients on both sides, we obtain

$$
a_{m n}=\sum_{i=0, j=0}^{k+1, k+1}(-1)^{i}(-1)^{j}\left(\begin{array}{c}
k+1 \\
k+1-i
\end{array}\right)\left(\begin{array}{c}
k+1 \\
k+1-j
\end{array}\right) S_{m-k, m-j,}^{(k)}
$$

where for the sake of uniformity we have set

$$
S_{p q}^{(k)}=0
$$

$(p$ or $q \leqq 0)$. 
Finally we have from the definition of $A_{m n}^{(k)}$

and hence

$$
\lim _{m, n=\infty} \frac{1_{m n}^{(k)}}{m_{m n}^{k} n^{k}}=\left\{\begin{array}{c}
1 \\
\{\Gamma(k+1)\}^{2},
\end{array}\right.
$$

$$
A_{m n}^{(i)}<. M m^{k} n^{k}
$$

$(m, n=1,2,3, \cdots)$,

where $\boldsymbol{M}$ is a positive constant.

It is evident that if our definition of summability is to be of wide use, it must be consistent with the more fundamental definition of convergence. That is to say, if a double series is convergent in accordance with the definition of Pringshem, it should be summable $(C k)$ for any $k>0$, and to the same value. We are naturally led to suspect that our definition will satisfy this requirement on account of its analogy to CEsìno's definition, which is known to satisfy a similar requirement. We shall find that it does so for a very wide class of convergent double series, namely for those for which

$$
\left|s_{m n}\right|=\left|\begin{array}{l}
S_{m n}^{(0)} \\
A_{m n}^{(0)}
\end{array}\right|<C \quad(m, n=1,2,3, \cdots),
$$

where $C$ is a positive constant. Moreover, when the condition (12) is satisfied for a convergent double series, not only will the ratio $S_{(m n}^{k} / A_{m n}^{(k)}$ approach the same value as $s_{m n}$ when $m$ and $n$ become infinite, but also it will have in common with $s_{m n}$ the property expressed by (12), namely of remaining less in absolute value than the positive constant $C$ for all values of $m$ and $n$.

In order to avoid any inconsistency between the theory of summability and that of convergence, or in the theory of summability itself, we will restrict ourselves to the consideration of double series for which

and at the same time

$$
\lim _{m, n=\infty} \frac{S_{m n}^{(k)}}{A_{m n}^{(k)}} \text { exists, }
$$

$$
\left|\frac{S_{m n}^{(k)}}{A_{m n}^{(k)}}\right|<C \quad(m, n=1,2,3, \cdots),
$$

where $C$ is a positive constant, for $k$ equal to zero or some positive integer.

It is possible that some or all of this restriction might be removed. We have not attempted to settle that question in the present paper for two reasons: first, because the series whose summability we proposed to investigate, namely the double Fourier's series, satisfies the restriction; secondly, because in the theorems about convergence factors that we needed for the applications, the same restriction naturally presented itself. However, from the standpoint of a general theory of summability for double series, it is desirable that the necessity of the restriction, or part of the restriction, should be investigated. 
If it should turn out that the condition (14) was necessary for consistency in our definitions, it would seem preferable to define the order of summability of a series as the lowest value of $k$ for which both (13) and (14) are satisfied. This, however, would necessitate either imposing the condition (12) in our definition of convergence, or having summability of order zero to agree with convergence only for the class of series for which (12) is satisfied.

Before proceeding to the discussion of the theorem of consistency, we wish to introduce a notation that we shall have occasion to use frequently in this paper, and which we shall use for the first time in the course of establishing the first of two lemmas needed in the proof of the theorem of consistency. The object of this notation is to furnish an abbreviation for certain expressions formed from a double sequence of quantities by selecting a square array of terms from the sequence, attaching to the terms certain binomial coefficients with signs that are alternately plus and minus, and taking the sum of all the resulting quantities. The most general form of such an expression is the following:

$$
\sum_{r=0, s=0}^{k+1, k+1}(-1)^{r}(-1)^{s}\left(\begin{array}{c}
k+1 \\
k+1-r
\end{array}\right)\left(\begin{array}{c}
k+1 \\
k+1-s
\end{array}\right) f_{i+r, j+s .}
$$

It will be seen that this expression bears to the double sequence $f_{i j}$ $(i, j=1,2,3, \cdots)$, a relation entirely analogous to that borne to a simple sequence by certain expressions known as $(k+1)$ th differences of such a sequence. Hence we may refer to expressions of the form (15) as the $(k+1)$ th differences of the corresponding double sequences. Moreover, we will adopt for such expressions a notation analogous to that used for differences of an ordinary sequence; this notation is completely defined by the following expression which represents the quantity (15),

$$
\Delta_{k+1}^{k+1} f_{i j} \text {. }
$$

We are now ready to prove the two lemmas needed in the proof of the theorem of consistency.

LEMMa 1. If we have two double sequences

such that

$$
a_{m n}, \quad b_{m n} \quad(m, n=1,2,3, \cdots),
$$

$$
\left.\left|\frac{\Delta_{1}^{1} a_{m n}}{\Delta_{1}^{1} b_{m n}}\right|<C \quad(m, n=0,1,2,3, \cdots)\right)^{*}
$$

where $C$ is a positive constant,

(b)

$$
\Delta_{1}^{1} b_{m n}>0 \quad(m, n=0,1,2,3, \cdots),
$$

* In forming $\Delta_{1}^{1} a_{m n}$ and $\Delta_{1}^{1} b_{m n}$ for $m$ or $n=0$, we put for the sake of uniformity

$$
a_{m n}=0=b_{m n}
$$

$(m$ or $n=0)$. 
$(c)$

$$
\begin{gathered}
\lim _{m, n=\infty} b_{m n}=\infty, \\
\lim _{p=\infty} \frac{b_{\mu q}}{b_{p q}}=0, \quad \lim _{q=\infty} \frac{b_{p v}}{b_{p q}}=0 \quad(\mu, \nu=1,2,3, \cdots),
\end{gathered}
$$

where the limits $(d)$ are approached uniformly, the first for all positive integral values of $q$, the second for all positive integral values of $p$,

then we shall have,

and furthermore

$$
\lim _{m, n=\infty} \frac{\Delta_{1}^{1} a_{m n}}{\Delta_{1}^{1} b_{m n}} \text { exists; }
$$

$$
\left|\frac{a_{m n}}{b_{m n}}\right|<C \quad(m, n=1,2,3, \cdots),
$$

will exist and be equal to the limit in (e).*

We will establish first the inequality (18). We have from conditions (a) and $(b)$

and hence

$$
\left|\Delta_{1}^{1} a_{m n}\right|<C \Delta_{1}^{1} b_{m n} \quad(m, n=1,2,3, \cdots),
$$

But obviously.

$$
\sum_{i=0, j=0}^{m-1, n-1}\left|\Delta_{1}^{1} a_{i j}\right|<C \sum_{i=0, j=0}^{m-1, n-1} \Delta_{1}^{1} b_{i j} \text {. }
$$

$$
a_{m n}=\sum_{i=0, j=0}^{m-1, n-1} \Delta_{1}^{1} a_{i j}, \quad b_{m n}=\sum_{i=0, j=0}^{m-1, n-1} \Delta_{1}^{1} b_{i j},
$$

so that, in view of condition (b) and (20), we have

$$
\left|\frac{a_{m n}}{b_{m n}}\right| \sum \frac{\sum_{0,0}^{m-1, n-1}\left|\Delta_{1}^{1} a_{i j}\right|}{\sum_{0,0}^{m-1, n-1} \Delta_{1}^{1} b_{i j}}<C \quad(m, n=1,2,3, \cdots) .
$$

It now remains to be shown that the limit (19) exists and is equal to the limit in $(e)$.

Since the limit in (e) exists, we can find a $\mu$ and $\nu$ corresponding to any arbitrarily small, positive quantity $\epsilon$, such that

$$
l-\epsilon<\frac{\Delta_{1}^{1} a_{m n}}{\Delta_{1}^{1} b_{m n}}<l+\epsilon \quad\left(\begin{array}{c}
m \geqq \mu \\
n \geqq \nu
\end{array}\right),
$$

where $l$ is the value of the limit in (e). Hence, in view of condition (b), it

* This lemma is a generalization to double sequences of a theorem for ordinary sequences due to Stouz. Cf. Bromwich, Infinite Series, p. 378. 
follows that

$$
(l-\epsilon) \Delta_{1}^{1} b_{m n}<\Delta_{1}^{1} a_{m n}<(l+\epsilon) \Delta_{1}^{1} b_{m n} \quad\left(\begin{array}{c}
m \geqq \mu \\
n \geqq \nu
\end{array}\right) .
$$

If we add the inequalities of the form (22) for all pairs of values of $m$ and $n$ such that $\mu \overline{\overline{ }} m \overline{\overline{ }} p, \nu \bar{\gtrless} n \overline{\bar{\gamma}} q$, we obtain

$$
\begin{aligned}
(l-\epsilon)\left(b_{p q}-b_{\mu q}-b_{p \nu}+b_{\mu \nu}\right)<a_{p q}- & a_{\mu q}-a_{p \nu}+a_{\mu \nu} \\
& <(l+\epsilon)\left(b_{p q}-b_{\mu q}-b_{p \nu}+b_{\mu \nu}\right) .
\end{aligned}
$$

If now we take $p$ and $q$ so large that $b_{p q}>0$, which we can evidently do in view of condition $(c)$, we can divide the last inequality through by $b_{p q}$. This gives us

$$
\begin{aligned}
(l-\epsilon)\left(1-\frac{b_{\mu q}}{b_{p q}}-\frac{b_{p \nu}}{b_{p q}}+\frac{b_{\mu \nu}}{b_{p q}}\right) & <\frac{a_{p q}}{b_{p q}}-\frac{a_{\mu q}}{b_{p q}}-\frac{a_{p v}}{b_{p q}}+\frac{a_{\mu \nu}}{b_{p q}} \\
& <(l+\epsilon)\left(1-\frac{b_{\mu q}}{b_{p q}}-\frac{b_{p v}}{b_{p q}}+\frac{b_{\mu \nu}}{b_{p q}}\right) .
\end{aligned}
$$

But from conditions $(c)$ and $(d)$ it follows that the limit

$$
\lim _{p, q=\infty}\left[1-\frac{b_{\mu q}}{b_{p q}}-\frac{b_{p \nu}}{b_{p q}}+\frac{b_{\mu \nu}}{b_{p q}}\right]
$$

exists and is equal to unity. Moreover, since,

$$
-\frac{a_{\mu q}}{b_{p q}}-\frac{a_{p v}}{b_{p q}}+\frac{a_{\mu \nu}}{b_{p q}}=-\frac{a_{\mu q}}{b_{\mu q}} \cdot \frac{b_{\mu q}}{b_{p q}}-\frac{a_{p v}}{b_{p v}} \cdot \frac{b_{p v}}{b_{p q}}+\frac{a_{\mu \nu}}{b_{p q}},
$$

it follows from conditions $(c)$ and $(d)$ and the inequality (21) that the limit

$$
\lim _{p, q=\infty}\left[-\frac{a_{\mu \nu}}{b_{p q}}-\frac{a_{p \nu}}{b_{p q}}+\frac{a_{\mu \nu}}{b_{p q}}\right]
$$

exists and is equal to zero. Hence we obtain from (23)

$$
l-\epsilon \overline{\lim } \frac{a_{p q}}{b_{p q}} \overline{\lim } \frac{a_{p q}}{b_{p q}} \bar{l} l+\epsilon .
$$

Since $\epsilon$ is an arbitrarily small positive quantity, it follows at once from the last inequality that

$$
\underline{\lim } \frac{a_{p q}}{b_{p q}}=\varlimsup \frac{a_{p q}}{b_{p q}}=l .
$$

Consequently the limit (19) exists and is equal to $l$, the value of the limit in (e). Our lemma is therefore proved.

Lemma 2. If the double series (1) is summable (Ck), and furthermore

$$
\left|\frac{S_{m n}^{(k)}}{A_{m n}^{(k)}}\right|<C \quad(m, n=1,2,3, \cdots),
$$


where $C$ is a positive constant, the scries will be summable $(C \overline{k+1})$ to the same value, and furthermore we shall have

$$
\left|\frac{S_{m n}^{(k+1)}}{A_{m n}^{(k+1)}}\right|<C \quad(m, n=1,2,3, \cdots) .
$$

From relations (6) and ( 7 ) it follows at once that

$$
\begin{aligned}
& \sum_{1,1}^{\infty} S_{m n}^{(k+1)} x^{m} y^{n} \sim\left(1+x+x^{2}+\cdots\right)\left(1+y+y^{2}+\cdots\right) \sum_{1,1}^{\infty} S_{m n}^{(k)} x^{m} y^{n}, \\
& \sum_{1,1}^{\infty} I_{m n}^{(k+1)} x^{m} y^{n} \sim\left(1+x+x^{2}+\cdots\right)\left(1+y+y^{2}+\cdots\right) \sum_{1,1}^{\infty} A_{m n}^{(k)} x^{m} y^{n},
\end{aligned}
$$

whence, on equating coefficients of corresponding terms, we have

$$
S_{m+1}^{(k+1)}=\sum_{1,1}^{m, n} S_{i j}^{(i)}, \quad A_{m n}^{(k+1)}=\sum_{i, 1}^{m, n} A_{i j}^{(i)} .
$$

From these two equations we obtain

$$
S_{m, n}^{(k)}=\Delta_{\mathrm{l}}^{1} S_{m-1, n-1}^{(k+1)}, \quad A_{m, n}^{(k)}=\dot{\Delta}_{\mathrm{l}}^{1} A_{m-1, n-1}^{(k+1)},
$$

where for the sake of uniformity we make use again of the notation (10).

We are now ready to apply Lemma 1 , taking for the first sequence in (17) the quantities $S_{m n}^{(k+1)}$, and for the second sequence the quantities $A_{m n}^{(k+1)}$. It follows at once from condition (24) of the present lemma, and equation (27), that condition ( $a$ ) of Lemma 1 is satisfied; that condition (b) is satisfied is an immediate consequence of the second equation in (27) and the definition of $A_{m n}^{(k)}$ (see equation (5)). It also follows without difficulty from the same definition that $(c)$ and $(d)$ of Lemma 1 are satisfied, and that condition $(e)$ is satisfied follows from the hypothesis of the present lemma that the double series (1) is summable $(C k)$.

Since all the conditions of Lemma 1 are satisfied by the quantities under consideration, it follows from that lemma that

exists and is equal to

$$
\lim _{m, n=\infty} \frac{S_{m n}^{(k+1)}}{A_{m n}^{(k+1)}}
$$

$$
\lim _{m, n=\infty} \frac{\Delta_{1}^{1} S_{m n}^{(k+1)}}{\Delta_{1}^{1}} A_{m n}^{(k+1)}=\lim _{m, n=\infty} \frac{S_{m n}^{(k)}}{A_{m n}^{(k)}},
$$

that is that the series (1) is summable $(C \overline{k+1})$ and to the same value to which it is summable $(C k)$.

It also follows from Lemma 1 that

$$
\left|\frac{S_{m n}^{(k+1)}}{A_{m n}^{(k+1)}}\right|<C \quad(m, n=1,2,3, \cdots) .
$$

The present lemma is, therefore, completely established. 
We are now prepared to establish the theorem of consistency which may be stated as follows:

Theorem I. If the series (1) is summable $(C k)$, where $k$ is zero or any positive integer, and if furthermore

$$
\left|\frac{S_{m n}^{(k)}}{A_{m n}^{(k)}}\right|<C \quad(m, n=1,2,3, \cdots),
$$

where $C$ is a positive constant, then the series will be summable $\left(C k^{\prime}\right)$, where $k^{\prime}$ is any integer greater than $k$, and to the same value, and furthermore we shall have

$$
\left|\frac{S_{m n}^{\left(k^{\prime}\right)}}{A_{m n}^{\left(k^{\prime}\right.}}\right|<C \quad(m, n=1,2,3, \cdots) .
$$

If $k^{\prime}-k=1$, this theorem reduces to Lemma 2. If $k^{\prime}-k=r>1$, the theorem follows at once from $r$ successive applications of Lemma 2.

Before closing this section we wish to define uniform convergence and uniform summability of double series, and point out a few facts in this connection. If the terms of the series (1) are functions of $x$ and $y$ throughout a certain region $R$ of the $x, y$-plane, and furthermore $s_{m n}$, defined by equation (3), approaches a limit uniformly for all values of $x$ and $y$ in $R$ as $m$ and $n$ become infinite, we shall say that the double series (1) is uniformly convergent. The definition for any other number of variables is entirely analogous. Similarly we define the series to be uniformly summable $(C k)$ in $R$ if the quotient (4) approaches a limit uniformly for all values of $x$ and $y$ in $R$, as $m$ and $n$ become infinite.

It is easy to show that if the terms of a double series are continuous throughout a region in which the series is uniformly convergent or uniformly summable, the function which the series represents will be continuous. The proof is entirely analogous to the well-known proof for simple series that a uniformly convergent series of continuous functions defines a continuous function. Moreover, it is easy to establish a test for the uniform convergence of a double series analogous to the well-known test of Weierstrass for the uniform convergence of a simple series, namely to show that if the general term of a double series is less in absolute value than the general term of a convergent double series of positive constants, throughout a certain region, the given double series will converge uniformly throughout that region.

\section{§2. Convergence Factors in Double Series.}

Before entering on the subject matter of this section we wish to define one further notation that we introduce for the sake of brevity. In analogy with the abbreviated notation introduced to represent the expression (15), we will set

$$
\Delta_{k+1, q}^{k+1, p} f_{i j}=\sum_{r=0, \ell=0}^{k+1-q, k+1-p}(-1)^{r}(-1)^{s}\left(\begin{array}{c}
k+1 \\
k+1-r
\end{array}\right)\left(\begin{array}{c}
k+1 \\
k+1-s
\end{array}\right) f_{i+r, j+s .}
$$

Trans. Am. Math. Soc. 6 
It will be observed that the expression on the right-hand side of (28) is equal to the expression (15) with certain of its terms suppressed. The number of terms suppressed and the ones which are suppressed, are indicated by the second upper and lower indices added to the expression (16) to form the lefthand side of (28). For the cases in which $p$ or $q$ is equal to $k+1$ we shall adopt a simpler way of writing the left-hand side of $(28)^{*}$; thus we shall set

$$
\Delta_{k+1, q}^{k+1, k+1} f_{i j}=\Delta_{k+1, q} f_{i j}, \quad \Delta_{k+1, k+1}^{k+1, p} f_{i j}=\Delta^{k+1, p} f_{i j} .
$$

This notation having been explained, we are ready to prove the theorems on convergence factors that we shall consider in this paper. We begin by considering the case of convergence factors in double series that are summable $(C 1)$. The results that we shall establish.for this case are special cases of the results that we shall obtain later on for the case of convergence factors in series summable $(C k)$. However, as these special cases are the ones that we shall make use of in the applications of this paper, it seems worth while to prove them separately, especially as the proof is considerably simpler than the proof of the general case.

Lemma 3. If the double series (1) is summable ( $C 1)$, and moreover

$$
\left|\frac{S_{m n}^{(1)}}{A_{m n}^{(1)}}\right|<C \quad(m, n=1,2,3, \cdots),
$$

where $C$ is a positive constant, then for all positive values of $\alpha$ and $\beta$ the series

$$
\sum_{m=1, n=1}^{\infty, \infty} a_{m n} f_{m n}(\alpha, \beta)
$$

will converge and have the same value as the series

$$
\sum_{i=1, j=1}^{\infty, \infty} S_{i j}^{(1)} \Delta_{2}^{2} f_{i j}(\alpha, \beta)
$$

which will also be convergent, provided the convergence factors $f_{i j}(\alpha, \beta)$ satisfy the following conditions:

$$
\begin{aligned}
\sum_{i=1, j=1}^{\infty} i j\left|\Delta_{2}^{2} f_{i j}(\alpha, \beta)\right|<K, \text { a positive constant } & (\alpha, \beta>0), \\
\lim _{m=\infty} m \sum_{j=1}^{\infty} j\left|f_{m j}(\alpha, \beta)\right|=0 & (\alpha, \beta>0), \\
\lim _{n=\infty} n \sum_{i=1}^{\infty} i\left|f_{i n}(\alpha, \beta)\right|=0 & (\alpha, \beta>0), \\
\lim _{m, n=\infty}\left[m n f_{m n}(\alpha, \beta)\right]=0 & (\alpha, \beta>0),
\end{aligned}
$$

\footnotetext{
* If either $p$ or $q$ is equal to zero, the zero value will he omitted.
} 
it being understood of course that the limiting processes indicated in conditions (a), (b), (c), and (d), all have a meaning.

Substituting in the expression

$$
\sum_{1,1}^{m, n} a_{i j} f_{i j}
$$

the value of $a_{i j}$ given by putting $k=1$ in (9), and rearranging the terms according to suffixes of $S$, we obtain the expression *

$$
\begin{aligned}
\sum_{1,1}^{m-2, n-2} S_{i j}^{(1)} \Delta_{2}^{2} f_{i j} & +\sum_{i=1}^{i=m-2} S_{i, n-1}^{(1)} \Delta_{2}^{2,1} f_{i, n-1} \\
& +\sum_{i=1}^{i=m-2} S_{i n}^{(1)} \Delta_{2} f_{i n}+\sum_{j=1}^{j=n-2} S_{m-1, j}^{(1)} \Delta_{2,1}^{2} f_{m-1, j} \\
& +\sum_{j=1}^{j=n-2} S_{m j}^{(1)} \Delta^{2} f_{m j}+S_{m-1, n-1}^{(1)} \Delta_{2,1}^{2,1} f_{m-i, n-1} \\
& +S_{m n}^{(1)} f_{m n}+S_{m-1, n}^{(1)} \Delta_{2,1} f_{m-1, n}+S_{m, n-1}^{(1)} \Delta^{2,1} f_{m, n-1}
\end{aligned}
$$

We shall show that for positive values of $\alpha$ and $\beta$, each term of the expression (34) approaches a limit as $m$ and $n$ become infinite, and that every term but the first approches the limit zero. From this it will follow that the series (31) converges for all positive values of $\alpha$ and $\beta$ and to the same limit as the series

* The simplest way to see that the expression (34) is identically equal to the expression (33) is the following: On expanding the terms of (33), we observe that each term of the double summation in (34) is obtained by selecting according to a fixed rule one term out of each of the expansions of nine terms of (33), these terms of (33) forming a square array whose upper left hand corner is the term with subscripts $p$ and $q$, in the case where we are forming the term in the double summation of (34) whose subscripts are $p$ and $q$. It is easily seen in this way that all the terms in the double summation of (34) can be obtained from the expansions of the terms of (33), and that this process uses up all the terms in the expansions of the $(m-2)(n-2)$ terms of (33) lying in a rectangle $(m-2)$ terms high and $(n-2)$ terms broad whose upper left-hand corner is the first term of (33), and some of the terms in the expansions of each of the other terms. It remains to verify the fact that the remaining terms in the expansions of the terms of (33) are identically equal to the terms in (34) after the first. This is easily seen by selecting terms from the expansions according to the same rule by which the terms of the double summation of (34) were obtained. It is obvious that such a process will give us incomplete terms of the form of the terms in this double summation, and that the degree of incompleteness depends on the position with regard to the edges of the rectangle of $m n$ terms formed by (33), of the term in whose expansion we select the first term to form the term of (34). The terms of (34) whose first terms are taken from one of the terms in the first $(m-2)$ terms of the $(n-1)$ th column in the double summation (33) form the first single summation in (34); the terms of (34) whose first terms are taken from one of the first $(m-2)$ terms of the $n$th calumn of (33) form the second single summation of (34), and so on. By accounting for all terms whose first term comes from the expansion of one of the terms of (33) that lie in the last two rows, the last two columns, or both, we get all the terms of (34) and we use up all the terms in the expansions of the terms of (33).

An entirely similar process will enable us to make the more complicated reduction required later on to reduce (33) to (49). 
(32) whose convergence will be established in proving that the first term in (34) approaches a limit as $m$ and $n$ become infinite. Thus our lemma will be completely established.

Consider first the first term in (34). This term is the sum of the $(m-2)(n-2)$ terms of the series (32) contained in a rectangle $(m-2)$ terms high and $(n-2)$ terms broad, taken from the upper left-hand corner of that series. In view of (30), we have for the general term of (32)

$$
\left|S_{m n}^{(1)} \Delta_{2}^{2} f_{m n}\right|<C m n\left|\Delta_{2}^{2} f_{m n}\right|\left(\begin{array}{c}
m, n=1,2,3, \cdots \\
\alpha, \beta>0
\end{array}\right) .
$$

But it follows from condition $(a)$ that the series whose general term is the expression on the right-hand side of (35) is convergent. Hence the series (32) is absolutely convergent, and consequently the first term in (34) apapproaches a limit as $m$ and $n$ become infinite. That the sixth, seventh, eighth, and ninth terms approach zero as $m$ and $n$ become infinite is an immediate consequence of conditions (30) and $(d)$. It remains to show that the same is true of the second, third, fourth, and fifth terms.

The second term of (34) is in absolute value less than or equal to

$$
\begin{aligned}
& \sum_{i=1}^{m-2}\left|S_{i, n-1}^{(1)} f_{i, n-1}\right|+2 \sum_{i=1}^{m-2}\left|S_{i, n-1}^{(1)} f_{i+1, n-1}\right|+\sum_{i=1}^{m-2}\left|S_{i, n-1}^{(1)} f_{i+2, n-1}\right| \\
& +2 \sum_{i=1}^{m-2}\left|S_{i, n-1}^{(1)} f_{i n}\right|+4 \sum_{i=1}^{m-2}\left|S_{i, n-1}^{(1)} f_{i+1, n}\right|+2 \sum_{i=1}^{m-2}\left|S_{i, n-1}^{(1)} f_{i+2}, n\right|,
\end{aligned}
$$

which in view of $(30)$ is in turn less than

$$
\begin{array}{r}
C(n-1) \sum_{i=1}^{m-2} i\left|f_{i, n-1}\right|+2 C(n-1) \sum_{i=1}^{m-2} i\left|f_{i+1, n-1}\right|+C(n-1) \sum_{i=1}^{m-2} i\left|f_{i+2, n-1}\right| \\
+2 C(n-1) \sum_{i=1}^{m-2} i\left|f_{i n}\right|+4 C(n-1) \sum_{i=1}^{m-2} i\left|f_{i+1, n}\right|+2 C(n-1) \sum_{i=1}^{m-2} i\left|f_{i+2, n}\right| \\
<4 C(n-1) \sum_{i=1}^{m} i\left|f_{i, n-1}\right|+8 C(n-1) \sum_{i=1}^{m} i\left|f_{i n}\right| .
\end{array}
$$

It follows at once from condition (c) that the expression on the right-hand side of the last inequality approaches zero as $m$ and $n$ become infinite. Hence the second term of (34), which is always less in absolute value than this expression, must also approach zero as a limit.

Consider now the third term of (34). It is obviously in absolute value less than or equal to

$$
\sum_{i=1}^{m-2}\left|S_{i n}^{(1)} f_{i n}\right|+2 \sum_{i=1}^{m-2}\left|S_{i n}^{(1)} f_{i+1, n}\right|+\sum_{i=1}^{m-8}\left|S_{i n}^{(1)} f_{i+2, n}\right|,
$$


which in view of (30) is in turn less than

$$
C n \sum_{i=1}^{m-2} i\left|f_{i n}\right|+2 C n \sum_{i=1}^{m-2} i\left|f_{i+1, n}\right|+C n \sum_{i=1}^{m-2} i\left|f_{i+2, n}\right|<4 C n \sum_{i=1}^{m} i\left|f_{i n}\right| .
$$

It follows at once from condition (c) that the expression on the right-hand side of the last inequality approaches zero as $m$ and $n$ become infinite. Hence the third term of (34), which is always less in absolute value than this expression, must also approach zero as a limit.

That the fourth and fifth terms of the expression (34) approach zero as a limit as $m$ and $n$ become infinite, may be shown by a discussion analogous to that used in the case of the second and third terms, the principal difference being that condition $(b)$ replaces condition $(c)$.

Thus we see that every term in (34) approaches a limit as $m$ and $n$ become infinite and that every term but the first approaches the limit zero. Hence, as we have pointed out before, our lemma is completely established.

We are now ready to prove the following theorem:

Theorem II.* If the double series (1) satisfies the conditions of Lemma 3, and the convergence factors $f_{i j}(\alpha, \beta)$ satisfy the conditions of that lemma and the following further conditions

$$
\begin{array}{r}
f_{i j}(\alpha, \beta) \text { is continuous is } \alpha \text { and } \beta \quad\left(\begin{array}{c}
i, j=1,2,3, \cdots \\
\alpha, \beta>0
\end{array}\right), \\
\lim _{\alpha, \beta=+0}\left[f_{i j}(\alpha, \beta)\right]=f_{i j}(0,0)=1 \quad(i, j=1,2,3, \cdots),
\end{array}
$$

then the series (31) will define a function of $\alpha$ and $\beta, F(\alpha, \beta)$, that is continuous for all positive values of $\alpha$ and $\beta$, and for which

$$
\lim _{\alpha, \beta=+0}[F(\alpha, \beta)]=S,
$$

where $S$ is the value of the series (1).

We know from Lemma 3 that under the conditions of that lemma the series (31) converges to the same value as the series (32) for all positive values of $\alpha$ and $\beta$. Hence if we can show that under the conditions of the present theorem the series (32) defines a function of $\alpha$ and $\beta$ that is continuous for all positive values of $\alpha$ and $\beta$ and for which (36) is true, our theorem will be proved.

If we set

$$
\frac{S_{m n}^{(1)}}{m n}=\sigma_{m n},
$$

* This theorem includes as a special case a theorem proved by Bromwich and Hardy. Cf. Proceedings of the London Mathematical Society, $2 d$ ser., vol. 2 (1905), p. 161. In the same paper the authors consider series summable by higher means, but the means used are the analogues of HörDer's means for simple series and not of Crsiro's. 
we shall have

Hence if we put

it will follow that

$$
\lim _{m, n=\infty}\left[\sigma_{m n}\right]=S
$$

$$
\sigma_{m n}=S+\epsilon_{m n}
$$

$$
\lim _{m, n=\infty}\left[\epsilon_{m n}\right]=0 .
$$

$\mathrm{By}$ means of (37) and (38) we can reduce the series (32) to the form

$$
S \sum_{1,1}^{\infty, \infty} i j \Delta_{2}^{2} f_{i j}(\alpha, \beta)+\sum_{1,1}^{\infty, \infty} i j \epsilon_{i j} \Delta_{2}^{2} f_{i j}(\alpha, \beta) .
$$

We can evaluate the series in the first term of (40) by applying Lemma 3 to the double series in which

$$
a_{11}=1, \quad a_{i j}=0
$$

( $i$ or $j>1$ ).

This series is obviously convergent, and moreover satisfies the restriction (12); hence by Theorem I it is summable $(C 1)$. It therefore follows from Lemma 3 that for this series

$$
\sum_{1,1}^{\infty, \infty} S_{i j}^{(1)} \Delta_{2}^{2} f_{i j}(\alpha, \beta)=\sum_{1,1}^{\infty, \infty} a_{i j} f_{i j}(\alpha, \beta) \quad(\alpha, \beta>0) .
$$

Making use of the values of the terms of the series (41) and the definition (2) of $S_{m n}^{(1)}$, we have for this series

$$
S_{i j}^{(1)}=i j \quad(i, j=1,2,3, \cdots) .
$$

by means of which equation (42) reduces to the form

$$
\sum_{1,1}^{\infty} i j \Delta_{2}^{2} f_{i j}(\alpha, \beta)=f_{11}(\alpha, \beta) \quad(\alpha, \beta>0) .
$$

From (44) it follows that the first term in (40) is equal to

$$
S f_{11}(\alpha, \beta) \text {. }
$$

This quantity is a continuous function of $\alpha$ and $\beta$ for all positive values of $\alpha$ and $\beta$ in view of condition (e), and from condition $(f)$ it is seen that it approaches $S$ as $\alpha$ and $\beta$ approach +0 . Since (40) is only another way of writing the series (32), it only remains to show that the second term of (40) is continuous for all positive values of $\alpha$ and $\beta$ and that it approaches zero as $\alpha$ and $\beta$ approach +0 . We will show this by proving that the terms of the series in the second term of (40) are continuous in the region

$$
\left(\begin{array}{c}
\alpha>0, \beta>0 \\
\alpha=0=\beta
\end{array}\right)
$$


that the series is uniformly convergent in that region, and that its value is zero for $\alpha=0=\beta$. It will then follow from one of the theorems referred to at the close of $\S 1$, namely from the theorem that a double series which is uniformly convergent in a region in which its terms are continuous, defines there a continuous function, that the second term of (40) has the desired properties. Thus our theorem will be completely established.

That the terms of the series in the second term of (40) are continuous in (45) follows at once from conditions $(e)$ and $(f)$. It remains to be shown then that the series is uniformly convergent in that region, and that it converges to zero for $\alpha=0=\beta$.

For $m$ and $n$ sufficiently large $\left|\epsilon_{i j}\right|$ becomes less than an arbitrarily small quantity $\epsilon$ by (39), and hence we have

$$
\left|\sum_{m, n}^{p, q} i j \epsilon_{i j} \Delta_{2}^{2} f_{i j}(\alpha, \beta)\right| \bar{\epsilon} \sum_{m, n}^{p, q} i j\left|\Delta_{2}^{2} f_{i j}(\alpha, \beta)\right|<K \epsilon \quad(\alpha, \beta>0),
$$

by (a), Lemma 3 .

For $\alpha=0=\beta$ each term of the series in the second term of (40) is identically zero, and hence the inequality (46) holds for those values also. Therefore the series just referred to converges uniformly throughout the region (45) and, moreover, it converges to zero for $\alpha=0=\beta$, since each term is identically zero for such values. We saw above that the terms of the series were continuous in (45), and hence, as we pointed out before, our theorem is completely established.

In Lemma 3 and Theorem II we have assumed that the series in which the convergence factors were introduced had constant terms. This limitation was made in order to avoid increasing the complexity of the formulæ and also in order to keep more clearly in mind the central fact of the theorem. Moreover, the extension to the case in which the terms of the series are functions of one or more variables is easily made. The most important fact in this connection is stated in the following corollary, where for the sake of definiteness and because that is the case of most interest in the applications, we restrict ourselves to series whose terms are functions of two variables.

Corollary. If the series

$$
\sum_{1.1}^{\infty, \infty} a_{i j}(x, y)
$$

is uniformly summable $(C 1)$ to the function $f(x, y)$ throughout a certain region $\boldsymbol{R}$, and moreover

$$
\left|\frac{S_{m n}^{(1)}(x, y)}{m n}\right|<C \quad(m, n=1,2,3, \cdots),
$$


where $C$ is a positive constant, then the series

$$
\sum_{1,1}^{\infty, \infty} a_{i j}(x, y) f_{i j}(\alpha, \beta)
$$

will converge for all positive values of $\alpha$ and $\beta$ and all values of $x$ and $y$ in $R$, and its value $F(x, y, \alpha, \beta)$ will approach $f(x, y)$ uniformly for all values of $x$ and $y$ in $R$, as $\alpha$ and $\beta$ approach +0 , provided the convergence factors $f_{i j}(\alpha, \beta)$ satisfy the conditions of Theorem $I I$.

The proof of this corollary requires only slight modifications in the reasoning of Lemma 3 and Theorem II.

We will next consider the case of convergence factors in series that are summable $(C k)$, where $k$ is zero or any positive integer. As in the special case just treated, we begin by proving a lemma.

Lemma 4. If the double series (1) is summable ( $C k)$, and moreover

$$
\left|\frac{S_{m n}^{(k)}}{A_{m n}^{(k)}}\right|<C \quad(m, n=1,2,3, \cdots),
$$

where $C$ is a positive constant, then the series (31) will converge for all positive values of $\alpha$ and $\beta$, and will have for such values of $\alpha$ and $\beta$ the same value as the series

$$
\sum_{i=1, j=1}^{\infty} S_{i j}^{(k)} \Delta_{k+1}^{k+1} f_{i j}(\alpha, \beta),
$$

which will also converge, provided the convergence factors $f_{i j}(\alpha, \beta)$ satisfy the follouing conditions

$$
\begin{array}{cc}
\sum_{i=1, j=1}^{\infty} i^{k} j^{k}\left|\Delta_{k+1}^{k+1} f_{i j}(\alpha, \beta)\right|<K \text { a positive constant } & (\alpha, \beta>0), \\
\lim _{m=\infty} m^{k} \sum_{j=1}^{\infty} j^{k}\left|f_{m j}(\alpha, \beta)\right|=0 & (\alpha, \beta>0), \\
\lim _{n=\infty} n^{k} \sum_{i=1}^{\infty} i^{k}\left|f_{i n}(\alpha, \beta)\right|=0 & (\alpha, \beta>0), \\
\lim _{m, n=\infty}\left[m^{k} n^{k} f_{m n}(\alpha, \beta)\right]=0 & (\alpha, \beta>0),
\end{array}
$$

it being understood that the limiting processes indicated in conditions $(a),(b),(c)$, and $(d)$, all have a meaning.

Substituting in (33) the value of $a_{i j}$ given by (9) and rearranging the terms according to suffixes of $S$, we obtain the expression *

* For the method of making the required computation see the footnote to Lemma 3. 


$$
\begin{aligned}
\sum_{1,1}^{m-k-1, n-k-1} S_{i j}^{(k)} \Delta_{k+1}^{k+1} f_{i j} & +\sum_{i=1, p=0}^{m-k-1, k} S_{i, n-k+p}^{(k)} \Delta_{k+1}^{k+1, p+1} f_{i, n-k+p} \\
& +\sum_{p=0, j=1}^{k, n-k-1} S_{m-k+p, j}^{(k)} \Delta_{k+1, p+1}^{k+1} f_{m-k+p, j} \\
& +\sum_{p=0}^{k} S_{m-k+p, n-k+p}^{(k)} \Delta_{k+1, p+1}^{k+1, p+1} f_{m-k+p, n-k+p} \\
& +\sum_{p=0}^{k-1}\left[\sum_{q=p+1}^{k} S_{m-k+p, n-k+q}^{(k)} \Delta_{k+1, p+1}^{k+1, q+1} f_{m-k+p, n-k+q}\right] \\
& +\sum_{p=0}^{k-1}\left[\sum_{q=p+1}^{k} S_{m-k+q, n-k+p}^{(k)} \Delta_{k+1, q+1}^{k+1, p+1} f_{m-k+q, n-k+p}\right] .
\end{aligned}
$$

We shall establish our lemma by showing that for $\alpha>0, \beta>0$ each of the terms in the above expression approaches a limit as $m$ and $n$ become infinite, and that every term but the first approaches the limit zero. Thus it will follow that the series (31) converges for $\alpha>0, \beta>0$, and to the same limit as the series (48), whose convergence will be established in proving that the first term of (49) approaches a limit as $m$ and $n$ become infinite.

Consider first the first term in (49). This term is the sum of the $(m-k-1)(n-k-1)$ terms of the series (48) contained in a rectangle $(m-k-1)$ terms high and $(n-k-1)$ terms broad, taken from the upper left-hand corner of that series. In view of (47) and (11), we have for the general term of (48)

$$
\left|S_{m n}^{(k)} \Delta_{k+1}^{k+1} f_{m n}\right|<C M m^{k} n^{k}\left|\Delta_{k+1}^{k+1} f_{m n}\right| \quad(\alpha, \beta>0) .
$$

But it follows from condition (a) that the series whose general term is the expression on the right-hand side of $(50)$ is convergent. IIence the series (48) is absolutely convergent, and consequently the first term of (49) approaches a limit as $m$ and $n$ become infinite, this limit being the value of the convergent series (48).

It remains now to be shown that the other terms in (49) approach the limit zero as $m$ and $n$ become infinite. The fourth, fifth, and sixth terms of this expression each consist of a fixed number of terms, that is a number dependent on $k$ but not on $m$ and $n$. Moreover each term is of the form

$$
N S_{i j} \dot{f}_{i+r, j+s}
$$

where $N$ is a coefficient that is never greater in absolute value than the largest coefficient in the expansion of $(a+b)^{k+1}(c+d)^{k+1}, i$ and $j$ are positive integers that become infinite with $m$ and $n$, and $r$ and $s$ are integers, each of which may have any one of the values $0,1,2, \cdots, k$. 
It follows easily from conditions (47) and $(d)$ that an expression of the form (51) approaches zero as a limit as $m$ and $n$ become infinite. For combining (47) with (11) we have

$$
\left|N S_{i j} f_{i+r, j+s}\right|<N C M i j\left|f_{i+r, j+s}\right| \overline{N C M}(i+r)(j+s)\left|f_{i+r, j+s}\right| \text {. }
$$

From condition $(d)$ and the fact that $i$ and $j$ are integers that become infinite with $m$ and $n$, it follows at once that the expression on the right-hand side of the last inequality approaches zero as $m$ and $n$ become infinite. Hence the expression on the left-hand side, and consequently the expression (51) approach zero as $m$ and $n$ become infinite. As the fourth, fifth, and sixth terms of (49) each consist of a fixed number of terms of the form (51), it follows that they also approach zero as $m$ and $n$ become infinite.

We have still to consider the second and third terms of (49). Here the number of terms in the summations is not fixed but varies with $m$ and $n$, and we have to make use of conditions $(b)$ and $(c)$ in order to show that the quantities we are discussing approach zero as $m$ and $n$ become infinite.

For the second term of (49) we have, in view of (47) and (11),

$$
\begin{array}{r}
\left|\sum_{i=1, p=0}^{m-k-1, k} S_{i, n-k+p}^{(k)} \Delta_{k+1}^{k+1, p+1} f_{i, n-k+p}\right| \sum \sum_{i=1, p=0}^{m-k-1, k}\left|S_{i, n-k+p}^{(k)}\right| \cdot\left|\Delta_{k+1}^{k+1, p+1} f_{i, n-k+p}\right| \\
<C M \sum_{i=1}^{m-k-1} i^{k}\left[\sum_{p=0}^{k}(n-k+p)^{k}\left|\Delta_{k+1}^{k+1, p+1} f_{i, n-k+p}\right|\right] .
\end{array}
$$

But, since

$$
\begin{aligned}
1+ & (k+1)+\frac{(k+1) k}{2 !}+\cdots+(k+1)+1 \\
& +(k+1)+(k+1)^{2}+\frac{(k+1)^{2} k}{2 !}+\cdots+(k+1)^{2}+(k+1) \\
& +\cdot \cdot \cdot \cdot \cdot \cdot \cdot \cdot \cdot \cdot \cdot \cdot \cdot \cdot \cdot \cdot \cdot \cdot \cdot \cdot \cdot \cdot \cdot \cdot \cdot \cdot \cdot \cdot \cdot+(k+1)^{2}+(k+1) \\
& +(k+1)+(k+1)^{2}+\frac{(k+1)^{2} k}{2 !}+\cdots+(k+1)+1=(1+1)^{k+1}(1+1)^{k+1}=4^{k+1},
\end{aligned}
$$

it is not difficult to see, if we write out the terms of the various expressions

$$
\Delta_{k+1}^{k+1, p+1} f_{i, n-k+p}
$$

and make use of the principle that the absolute value of the sum of several quantities is less than or equal to the sum of the absolute values, that

$$
\begin{aligned}
\sum_{p=0}^{k}(n-k+p)^{k} \mid \Delta_{k+1}^{k+1, p+1} & f_{i, n-k+p} \mid \\
& <4^{k+1} \sum_{p=0}^{k}(n-k+p)^{k}\left\{\sum_{q=0}^{k+1}\left|f_{i+q, n-k+p}\right|\right\} .
\end{aligned}
$$


Combining (52) and (53), we obtain

$$
\begin{aligned}
& \left|\sum_{i=1, p=0}^{m-k-1, k} S_{i, n-k+p}^{(k)} \Delta_{k+1}^{k+1, p+1} f_{i, n-k+p}\right| \\
& \quad<4^{k+1} C M \sum_{p=0}^{k}(n-k+p)^{k}\left[\sum_{i=1}^{m-k-1} i^{k}\left\{\sum_{q=0}^{k+1}\left|f_{i+q, n-k+p}\right|\right\}\right] .
\end{aligned}
$$

But

$$
\sum_{i=1}^{m-k-1} i^{k}\left\{\sum_{q=0}^{k+1}\left|f_{i+q, n-k+p}\right|\right\}<(k+2) \sum_{i=1}^{m} i^{k}\left|f_{i, n-k+p}\right|,
$$

and hence we have finally, from (54) and (55),

$$
\begin{aligned}
& \left|\sum_{i=1, p=0}^{m-k-1, k} S_{i, n-k+p}^{(k)} \Delta_{k+1}^{k+1, p+1} f_{i, n-k+p}\right| \\
& <(k+2) 4^{k+1} C M \sum_{p=0}^{k}(n-k+p)^{k}\left\{\sum_{i=1}^{m} i^{k}\left|f_{i, n-k+p}\right|\right\} .
\end{aligned}
$$

It follows without difficulty from condition $(c)$ that the right-hand member of (56) approaches zero as $m$ and $n$ become infinite. Consequently the lefthand member, that is to say the second term of (49), approaches zero as $m$ and $n$ become infinite.

By a discussion precisely analogous to the above we may obtain for the third term of (49) the inequality

$$
\begin{aligned}
& \left|\sum_{p=0, j=1}^{k, m-k-1} S_{m-k+p, j}^{(k)} \Delta_{k+1, p+1}^{k+1} f_{m-k+p, j}\right| \\
& <(k+2) 4^{k+1} C M \sum_{p=0}^{k}(m-k+p)^{k}\left\{\sum_{j=1}^{n} j^{k}\left|f_{m-k+p, j}\right|\right\} .
\end{aligned}
$$

By means of this inequality and condition $(b)$ we may infer at once that the third term of (49) approaches zero as $m$ and $n$ become infinite.

We have now shown that each term in (49) approaches a limit as $m$ and $n$ become infinite, and that every term but the first approaches the limit zero. Hence, as we have pointed out before, our lemma is completely established.

With the aid of Lemma 4 we can prove the following theorem:

Theorem III.* If the double series (1) satisfies the conditions of Lemma 4 . and the convergence factors $f_{i j}(\alpha, \beta)$ satisfy the conditions of that lemma and the following further conditions

$$
f_{i j}(\alpha, \beta) \text { is continuous in } \alpha \text { and } \beta\left(\begin{array}{c}
i, j=1,2,3, \cdots \\
\alpha, \beta>0
\end{array}\right) \text {, }
$$

* Theorem III is a generalization to the case of double series of a theorem for simple series due to Bromwich. Cf. Mathematische Annalen, vol. 65 (1908), p. 358. Recently BRomwicn's theorem has been generalized to the case of simple series whose order of summability is non-integral by Chapman and by Ottolenghi. Cf. Proce eding of the London Mathematical Society, ser. 2, vol. 9 (1911), p. 382, and Giornale di Matematichedi Battaglini, vol. 49 (1911), p. 245. 


$$
\lim _{a, \beta=+0}\left[f_{i j}(\alpha, \beta)\right]=f_{i j}(0,0)=1 \quad(i, j=1,2,3, \cdots),
$$

then the series (31) will define a function of $\alpha$ and $\beta, F(\alpha, \beta)$, that is continuous for all positive values of $\alpha$ and $\beta$, and for which

$$
\lim _{\alpha, \beta=+0}[F(\alpha, \beta)]=S,
$$

where $S$ is the value of the series (1).

The above theorem is proved with the aid of Lemma 4 in almost precisely the same manner that Theorem II was proved with the aid of Lemma 3. Practically the only changes in the wording of the proof which are not merely those of notation consist in the substitution for the equality (43), of the equality

$S_{i j}^{(k)}=\sum_{p=1, q=1}^{i, j}\left(\begin{array}{c}k+i-p-1 \\ k-1\end{array}\right)\left(\begin{array}{c}k+i-q-1 \\ k-1\end{array}\right)=A_{i j}^{(k)} \quad(i, j=1,2,3, \cdots)$,

which follows from (2) and (8), and the use of the inequality (11) in proving the uniform convergence of the series that corresponds to the series in the second term of $(40)$.

A corollary to the present theorem that bears the same relation to it that the corollary to Theorem II does to that theorem may be established without difficulty.

\section{§3. The Summability of the Double Fourier's Series at Points of Continuity of the Function Developed.*}

We will begin this section by stating that wherever we refer to a function as being finite and integrable, the statement is to be taken in the sense that the function has an integral according to the definition of LEBESGUE.

We will now proceed to prove several lemmas that are needed in the proof of our principal theorem.

Lemma 5. Let $R$ be a region in the $\alpha, \beta$-plane lying within the square whose

* The convergence of the double Fourier's series has been studied by KraUse, HARDY, Veraerio, and W. H. Young. Cf. Young's memoir in the Proce eding of the L ond on Mathematical Society, (ser. 2, vol. 11 (1912), p. 133), where reference to the other writers is made. In the paper just cited, Young has also considered the summability, and has obtained the result stated in Theorem IV of this section. The present paper was completed and sent to editors some time before the appearance of YounG's paper, and the results of this section were indicated in an abstract of part of the paper published in-the Bulletin of the American Mathematical Society (vol. 18 (1912), p. 223), also before the appearance of Young's paper. The writer has also studied the summability of the double Fourier's series at points of discontinuity ii the function developed, as is indicated in the abstract just referred to and in a note published in the Co m tes h e n d u s (vol. 154 (1912), p. 126). The results obtained in this connection will be incorporated in another paper. 
sides are $\alpha= \pm\left(\pi-\rho_{1}\right), \beta= \pm\left(\pi-\rho_{1}\right)$ where $\rho_{1}$ is a small positive quantity, and such that no point of $R$ lies within the circle whose center is at the origin and whose radius is $\rho_{2}$, where $\rho_{2}$ is another small positive quantity. Then, if $\varphi(\alpha, \beta)$ is a function that is finite and integrable in the region $R$, the limit

$$
\lim _{m, n=\infty}\left[\frac{1}{m n} \iint_{R} \varphi(\alpha, \beta) \frac{\sin ^{2} m \alpha}{\sin ^{2} \alpha} \cdot \frac{\sin ^{2} n \beta}{\sin ^{2} \beta} d \alpha d \beta\right]
$$

will exist and be equal to zero.

Let us represent by $M$ the upper limit of the absolute value of $\varphi(\alpha, \beta)$ in the region $R$, and by $\rho$ the smaller of the two positive quantities $\rho_{1}$ and $\rho_{2} / \sqrt{2}$. Let $\epsilon$ be a given positive quantity, arbitrarily small, and let $q$ be a positive integer such that

$$
\frac{4 \pi^{2} M}{q \sin ^{2} \rho}<\frac{\epsilon}{2} .
$$

We will show that for value of $m$ and $n$ greater than $q$, the absolute value of the expression in brackets in (58) is less than $\epsilon$. Thus our lemma will be established.

Let us divide the region $R$ into two parts, $R_{1}$ and $R_{2}$, such that $R_{1}$ contains all the points for which $|\alpha|<\rho_{2} / \sqrt{2}$, and $R_{2}$ contains all the points for which $|\alpha| \overline{\rangle} \rho_{2} / \sqrt{2}$. It may happen that there are no points such that $|\alpha|<\rho_{2} / \sqrt{2}$; in that case $R_{2}$ will coincide with $R$. For any point of $R$ we have from the conditions of our lemma.

and since for the points of $R_{1}$

$$
\alpha^{2}+\beta^{2} \overline{>} \rho_{2}^{2},
$$

$$
\alpha^{2}<\frac{\rho_{2}^{2}}{2},
$$

it follows on subtracting the second inequality from the first that

$$
\beta^{2}>\frac{\rho_{2}^{2}}{2} \overline{>} \rho^{2}
$$

for all points in $R_{1}$. We have then

$$
\begin{aligned}
& \left|\frac{1}{m n} \iint_{k_{1}} \varphi(\alpha, \beta) \frac{\sin ^{2} m \alpha}{\sin ^{2} \alpha} \frac{\sin ^{2} n \beta}{\sin ^{2} \beta} d \alpha d \beta\right| \\
& \overline{\bar{k}} \frac{1}{m n} \iint_{R_{1}}|\varphi(\alpha, \beta)| \frac{\sin ^{2} m \alpha}{\sin ^{2} \alpha} \frac{\sin ^{2} n \beta}{\sin ^{2} \beta} d \alpha d \beta \\
& <\frac{M}{m n \sin ^{2} \rho} \iint_{R_{1}} \frac{\sin ^{2} m \alpha}{\sin ^{2} \alpha} d \alpha d \beta \text {. }
\end{aligned}
$$


But

$$
\frac{1}{m} \iint_{R_{1}} \frac{\sin ^{2} m \alpha}{\sin ^{2} \alpha} d \alpha d \beta<\frac{1}{m} \int_{-\pi}^{\pi} \int_{-\pi}^{\pi} \frac{\sin ^{2} m \alpha}{\sin ^{2} \alpha} d \alpha d \beta=4 \pi^{2},
$$

a result which follows easily from the relation

$$
\frac{\pi}{2}=\frac{1}{n} \int_{0}^{\pi / 2} \frac{\sin ^{2} n \alpha}{\sin ^{2} \alpha} d \alpha
$$

established in Fejér's paper.* Hence, combining (60), (61), and (59) we obtain

$$
\left|\frac{1}{m n} \iint_{R_{1}} \varphi(\alpha, \beta) \frac{\sin ^{2} m \alpha}{\sin ^{2} \alpha} \cdot \frac{\sin ^{2} n \beta}{\sin ^{2} \beta} d \alpha d \beta\right|<\frac{4 \pi^{2} M}{n \sin ^{2} \rho}<\frac{\epsilon}{2} \quad(n \geqq q) .
$$

If $R_{1}$ has an area equal to zero, it is obvious that (62) still holds.

Since for all points of $R_{2},|\alpha| \overline{>} \rho_{2} / \sqrt{2}$, we may obtain in an entirely similar manner

$$
\left|\frac{1}{m n} \iint_{R_{1}} \varphi(\alpha, \beta) \frac{\sin ^{2} m \alpha}{\sin ^{2} \alpha} \cdot \frac{\sin ^{2} n \beta}{\sin ^{2} \beta} d \alpha d \beta\right|<\frac{4 \pi^{2} M}{m \sin ^{2} \rho}<\frac{\epsilon}{2} \quad(m \geqq q) .
$$

Combining (62) and (63) we have

$$
\left|\frac{1}{m n} \iint_{R} \varphi(\alpha, \beta) \frac{\sin ^{2} m \alpha}{\sin ^{2} \alpha} \cdot \frac{\sin ^{2} n \beta}{\sin ^{2} \beta} d \alpha d \beta\right|<\epsilon \quad(m, n \geqq q),
$$

and as pointed out before, our lemma is established.

LemмA 6. If $h, h_{1}, k$, and $k_{1}$ are positive numbers less than $\pi$, the limit

$$
\lim _{m, n=\infty}\left[\frac{1}{m n \pi^{2}} \int_{-h_{1}}^{h} \int_{-k_{1}}^{k} \frac{\sin ^{2} m \alpha}{\sin ^{2} \alpha} \cdot \frac{\sin ^{2} n \beta}{\sin ^{2} \beta} d \alpha d \beta\right]
$$

will exist and be equal to unity.

The expression in brackets in (64) may be written in the form

(65) $\frac{1}{m n \pi^{2}} \int_{-\pi / 2}^{\pi / 2} \int_{-\pi / 2}^{\pi / 2} \frac{\sin ^{2} m \alpha}{\sin ^{2} \alpha} \cdot \frac{\sin ^{2} n \beta}{\sin ^{2} \beta} d \alpha d \beta \pm \frac{1}{m n} \iint_{R} \frac{1}{\pi^{2}} \cdot \frac{\sin ^{2} m \alpha}{\sin ^{2} \alpha} \cdot \frac{\sin ^{2} n \beta}{\sin ^{2} \beta} d \alpha d \beta$

where $R$ is used to represent the region that must be added to or subtracted from the square whose sides are $\alpha= \pm \frac{1}{2} \pi, \beta= \pm \frac{1}{2} \pi$, in order to produce the rectangle whose sides are $\alpha=h, \alpha=-h_{1}, \beta=k, \beta=-k_{1}$. It follows at once from Lemma 5 that the limit of the second term in (65), as $m$ and $n$ become infinite, is zero, and hence it only remains to determine the limit of the first term.

* L. c., p. 55. Also Carslaw, Introduction to the Theory of Fourier's Series and Integrals, etc., p. 142. In FEJtR's original paper there is a misprint in some of the formulæ on the page quoted, $\pi$ occurring instead of $\frac{1}{2} \pi$. 
That term may be written in the form

$$
\left\{\frac{1}{m \pi} \int_{-\pi / 2}^{\pi / 2} \frac{\sin ^{2} m \alpha}{\sin ^{2} \alpha} d \alpha\right\}\left\{\frac{1}{n \pi} \int_{-\pi / 2}^{\pi / 2} \frac{\sin ^{2} n \beta}{\sin ^{2} \beta} d \beta\right\}
$$

But we know from Fejer's work * that the value of this expression for any positive, integral values of $m$ and $n$ is unity. Hence its limit as $m$ and $n$ become infinite is unity, and since the limit of the second term in (65) is zero, it follows that the limit of (65), as $m$ and $n$ become infinite, is unity.

Lемма 7. Let $R$ be a region in the $\alpha, \beta$-plane lying within the square whose sides are $\alpha= \pm\left(\pi-\rho_{1}\right), \beta= \pm\left(\pi-\rho_{1}\right)$ where $\rho_{1}$ is a small positive quantity, and such that the point $\alpha=0, \beta=0$ lies within or on the boundary of $R$. Then if $\varphi(\alpha, \beta)$ is a function that is finite and integrable in $R$, the limit

$$
\lim _{m, n=\infty}\left\lfloor\frac{1}{m n} \iint_{R} \varphi(\alpha, \beta) \frac{\sin ^{2} m \alpha}{\sin ^{2} \alpha} \cdot \frac{\sin ^{2} n \beta}{\sin ^{2} \beta} d \alpha d \beta\right]
$$

will exist and be equal to zero, provided

$$
\lim _{a, \beta=0}[\varphi(\alpha, \beta)]=0
$$

In view of (67) we may choose $\rho_{2}$ so small that

$$
|\varphi(\alpha, \beta)|<\frac{\epsilon}{2 \pi^{2}} \quad\left(\alpha^{2}+\beta^{2}<\rho_{2}^{2}\right),
$$

where $\epsilon$ is an arbitrarily small, positive quantity. Let us divide $R$ into parts, $R_{1}$ and $R_{2}$, where $R_{1}$ is the circle with center at the origin and radius equal to $\rho_{2}$, or such part of that circle as is included in $R$, and $R_{2}$ is the rest of $R$. It follows at once from Lemma 5 that

$$
\lim _{m, n=\infty}\left[\frac{1}{m n} \iint_{R_{2}} \varphi(\alpha, \beta) \frac{\sin ^{2} m \alpha \alpha}{\sin ^{2} \alpha} \cdot \frac{\sin ^{2} m \beta}{\sin ^{2} \beta} d \alpha d \beta\right]=0,
$$

and hence we can choose $q$ so large that

$$
\left|\frac{1}{m n} \iint_{R_{2}} \varphi(\alpha, \beta) \frac{\sin ^{2} m \alpha}{\sin ^{2} \alpha} \frac{\sin ^{2} n \beta}{\sin ^{2} \beta} d \alpha d \beta\right|<\frac{\epsilon}{2} \quad(m, n \geqq q) .
$$

Moreover, in view of (68),

* See the previous footnote. 


$$
\begin{aligned}
\frac{1}{m n} \iint_{R_{1}} \varphi(\alpha, \beta) & \frac{\sin ^{2} m \alpha}{\sin ^{2} \alpha} \cdot \frac{\sin ^{2} n \beta}{\sin ^{2} \beta} d \alpha d \beta \\
& \overline{1} \frac{1}{m n} \iint_{R_{1}}|\varphi(\alpha, \beta)| \cdot \frac{\sin ^{2} m \alpha}{\sin ^{2} \alpha} \cdot \frac{\sin ^{2} n \beta}{\sin ^{2} \beta} d \alpha d \beta \\
& <\frac{\epsilon}{2 \pi^{2}}\left\{\frac{1}{m n} \iint_{R_{1}} \frac{\sin ^{2} m \alpha}{\sin ^{2} \alpha} \cdot \frac{\sin ^{2} n \beta}{\sin ^{2} \beta} d \alpha d \beta\right\} \\
& <\frac{\epsilon}{2 \pi^{2}}\left\{\frac{1}{m n} \int_{-\pi / 2}^{\pi / 2} \int_{-\pi / 2}^{\pi / 2} \frac{\sin ^{2} m \alpha}{\sin ^{2} \alpha} \cdot \frac{\sin ^{2} n \beta}{\sin ^{2} \beta} d \alpha d \beta\right\} \\
& =\frac{\epsilon}{2 \pi^{2}}\left[\frac{1}{m} \int_{-\pi / 2}^{\pi / 2} \frac{\sin ^{2} m \alpha}{\sin ^{2} \alpha} d \alpha\right] \cdot\left[\frac{1}{n} \int_{-\pi / 2}^{\pi / 2} \frac{\sin ^{2} n \beta}{\sin ^{2} \beta} d \beta\right]=\frac{\epsilon}{2} .^{*}
\end{aligned}
$$

Combining (69) and (70) we have

$$
\left|\frac{1}{m n} \iint_{R} \varphi(\alpha, \beta) \frac{\sin ^{2} m \alpha}{\sin ^{2} \alpha} \cdot \frac{\sin ^{2} n \beta}{\sin ^{2} \beta} d \alpha d \beta\right|<\epsilon \quad(m, n \geqq q),
$$

from which our lemma follows at once.

Remark.-In the three previous lemmas it was not found necessary to place any restriction on the behavior of the ratio of $m$ and $n$ as these two quantities become infinite. It may be noted further that the conclusions of these lemmas hold equally well if, instead of letting $m$ and $n$ become infinite together, we let $m$ become infinite first, holding $n$ fixed, and then let $n$ become infinite, or vice versa. We have not insisted on this latter point in the proofs of the lemmas, because it is not of much importance in the applications we have in view.

We are now ready to consider the summability of the development of a function of two variables, $f(x, y)$, in a double Fourier's series, that is the summability of the double series

$$
\begin{gathered}
\sum_{m=1, n=1}^{\infty, \infty} \frac{1}{2^{E(1 / n)+E(1 / n)} \pi^{2}} \int_{-\pi}^{+\pi} \int_{-\pi}^{\pi} f\left(x^{\prime}, y^{\prime}\right) P_{m n}\left(x, y, x^{\prime}, y^{\prime}\right) d x^{\prime} d y^{\prime} \\
P_{m n}\left(x, y, x^{\prime}, y^{\prime}\right)=\cos \left[(m-1)\left(x^{\prime}-x\right)\right] \cos \left[(n-1)\left(y^{\prime}-y\right)\right]
\end{gathered}
$$

$E(z)$ being used as in the theory of numbers to represent the largest integer contained in $z$.

ThEOREM IV. If the function $f(x, y)$ is finite and integrable in the region

$$
(-\pi \overline{<} x \bar{\Sigma} \pi,-\pi \overline{\overline{<}} y \overline{\overline{<}} \boldsymbol{\pi}),
$$

the development of the function in a double Fourier's series will be summable

* See the previous footnote. 
(C1) to the value of the function at every interior point of the region (73) at which the function is continuous.

We have for the series (71)*

$$
\begin{aligned}
\frac{S_{m n}^{(1)}(x, y)}{A_{m n}^{(1)}} & =\frac{S_{m n}^{(1)}(x, y)}{m n} \\
& =\frac{1}{4 m n \pi^{2}} \int_{-\pi}^{\pi} \int_{-\pi}^{\pi} f\left(x^{\prime}, y^{\prime}\right)\left(\frac{\sin \frac{m\left(x^{\prime}-x\right)}{2}}{\sin \frac{x^{\prime}-x}{2}}\right)^{2}\left(\frac{\sin \frac{n\left(y^{\prime}-y\right)}{2}}{\sin \frac{y^{\prime}-y}{2}}\right)^{2} d x^{\prime} d y^{\prime} .
\end{aligned}
$$

If we set

$$
x^{\prime}-x=2 \alpha, \quad y^{\prime}-y=2 \beta,
$$

we obtain

$$
\frac{S_{m n}^{(1)}(x, y)}{m n}=\frac{1}{m n \pi^{2}} \int_{-(\pi+x) / 2}^{(\pi-x) / 2} \int_{-(\pi+y) / 2}^{(\pi-y) / 2} f(x+2 \alpha, y+2 \beta) \frac{\sin ^{2} m \alpha}{\sin ^{2} \alpha} \cdot \frac{\sin ^{2} n \beta}{\sin ^{2} \beta} d \alpha d \beta
$$

If we can show that the expression on the right-hand side of (75) approaches $f(x, y)$ as a limit as $m$ and $n$ become infinite, at any interior point of the region (73) at which $f(x, y)$ is continuous, our theorem will be established.

Let us put

$$
\varphi(\alpha, \beta)=f(x+2 \alpha, y+2 \beta)-f(x, y) \text {. }
$$

The right-hand side of (75) may then be written in the form

$$
\begin{aligned}
& \frac{f(x, y)}{n m \pi^{2}} \int_{-(\pi+x) / 2}^{(\pi-x) / 2} \int_{-(\pi+y) / 2}^{(\pi-y) / 2} \frac{\sin ^{2} m \alpha}{\sin ^{2} \alpha} \cdot \frac{\sin ^{2} n \beta}{\sin ^{2} \beta} d \alpha d \beta \\
& \quad+\frac{1}{m n \pi^{2}} \int_{-(\pi+x) / 2}^{(\pi-x) / 2} \int_{-(\pi+y) / 2}^{(\pi-y) / 2} \varphi(\alpha, \beta) \frac{\sin ^{2} m \alpha}{\sin ^{2} \alpha} \cdot \frac{\sin ^{2} n \beta}{\sin ^{2} \beta} d \alpha d \beta .
\end{aligned}
$$

But from its definition $\varphi(\alpha, \beta)$ is finite and integrable in the region of integration of the integrals in (77), and moreover

$$
\lim _{\alpha, \beta=0}[\varphi(\alpha, \beta)]=0,
$$

provided $f(x, y)$ is continuous at the point $(x, y)$. It therefore follows from Lemma 7 that the second term in (77) approaches zero as a limit as $m$ and $n$ become infinite, provided $(x, y)$ is an interior point of the region (73) and $f(x, y)$ is continuous at that point. Moreover, it follows from Lemma 6 that the first term in (77), and therefore the whole expression (77), approaches as a limit $f(x, y)$ as $m$ and $n$ become infinite. But since (77) is equal to the right-hand side of (75), it follows that the limit of that expression as $m$ and $n$ become infinite is $f(x, y)$. Our theorem is, therefore, proved.

* This follows at once from the corresponding reduction for the ordinary Fourier's series. See Fejtr, 1. c., p. 54. Also Carglaw, 1. c., p. 141. 
Corollary 1. If $f(x, y)$ satisfies the conditions of Theorem IV, its Fourier's development will be uniformly summable to $f(x, y)$ throughout any region whose boundary is interior to the boundary of a region of continuity of $f(x, y)$.

If we make use of the principle that a function of two variables is uniformly continuous in any closed region in which it is continuous, it requires only a slight modification of the reasoning in Lemmas 5 and 7 and Theorem IV to establish this corollary.

Before stating the second corollary to Theorem IV we wish to introduce a definition which will serve to simplify somewhat its statement. If a double series has each of its rows summable $(C k)$, and the series formed from the values of the rows is summable ( $C k)$, we will say that the double series is summable $(C k)$ by rows, and we will ascribe to it the value of the series formed from the values of the rows. We define in a similar manner a double series summable ( Ck) by columns, and the value of such a series.

We are now ready to state the following corollary:

Corollary 2. If $f(x, y)$ satisfies the conditions of Theorem IV, its Fourier's development will be summable $(C 1)$ by rows or by columns at every interior point of the region (73) at which $f(x, y)$ is continuous, and to the value of the function.

The proof of this corollary * follows from the remark at the end of Lemma 7 in the same manner that the proof of Theorem IV follows from Lemmas 5, 6, and 7.

For the applications to mathematical physics we have in view, we need to know something about the behavior of the Fourier's development of $f(x, y)$ throughout the whole region (73) in cases where there are points of discontinuity of the function in the region. The necessary information is embodied in the following theorem:

Theorem V. If $f(x, y)$ satisfies the conditions of Theorem IV, we shall have for its Fourier's development

$$
L<\frac{S_{m n}^{(1)}(x, y)}{m n}<M\left(\begin{array}{c}
m, n=1,2,3, \cdots \\
-\pi \leqq x \leqq \pi,-\pi \leqq y \leqq \pi
\end{array}\right)
$$

where $M$ and $L$ are the upper and lower limits of $f(x, y)$ in the region (73).

In view of equation (75), we have

$$
\begin{aligned}
\frac{L}{m n \pi^{2}} \int_{-(\pi+x) / 2}^{(\pi-x) / 2} \int_{-(\pi+y) / 2}^{(\pi-y) / 2} \frac{\sin ^{2} m \alpha}{\sin ^{2} \alpha} \cdot \frac{\sin ^{2} n \beta}{\sin ^{2} \beta} d \alpha d \beta & \\
& <\frac{S_{m n}^{(1)}(x, y)}{m n}<M \int_{-(\pi+x) / 2}^{(\pi-x) / 2} \int_{-(\pi+y) / 2}^{(\pi-y) / 2} \frac{\sin ^{2} m \alpha}{\sin ^{2} \alpha} \cdot \frac{\sin ^{2} n \beta}{\sin ^{2} \beta} d \alpha d \beta .
\end{aligned}
$$

* We should note that this corollary is also an almost immediate consequence of FEJER's theorem about the summability of an ordinary Fourier's series, for it follows at once from two successive applications of that theorem. However, we cannot in this way obtain Theorem IV from Festr's theorem, for a double series summable by rows or columns is not necessarily summable as a double series, a fact that may be inferred at once from a theorem due to PrinasHEIM (cf. Münchener Berichte (1897), p. 107). 
But

$$
\begin{aligned}
\int_{-(\pi+x) / 2}^{(\pi-x) / 2} \int_{-(\pi+y) / 2}^{(\pi-y) / 2} \frac{\sin ^{2} m \alpha}{\sin ^{2} \alpha} \cdot \frac{\sin ^{2} n \beta}{\sin ^{2} \beta} d \alpha d \beta \\
=\left(\int_{-(\pi+x) / 2}^{(\pi-x) / 2} \frac{\sin ^{2} m \alpha}{\sin ^{2} \alpha} d \alpha\right)\left(\int_{-(\pi+y) / 2}^{(\pi-y) / 2} \frac{\sin ^{2} n \beta}{\sin ^{2} \beta} d \beta\right)=1, *
\end{aligned}
$$

and therefore our theorem follows at once.

\section{§4. Application.}

We will now illustrate the application of the above results to problems of mathematical physics by considering a problem in the flow of heat. Suppose we wish to determine at any instant the temperature of any point of a thin rectangular plate, whose perimeter is kept at the temperature zero and whose initial temperature we know. Let $b$ be the length and $c$ the breadth of the plate, and $f(x, y)$ be a function of two variables that gives us the initial temperature of the plate at each point when we take the lower left-hand corner of the plate as the origin and the sides of lengths $b$ and $c$ as $x$ and $y$ axes respectively. Then the ordinary, formal method of constructing a solution for problems of this type gives us for the temperature of any point at any instant $\dagger$

$$
\sum_{m=1, n=1}^{\infty, \infty} u_{m n}(x, y, t)=\frac{4}{b c} \sum_{m=1, n=1}^{\infty, \infty} a_{m n} e^{-a^{2} \pi^{2}\left(m^{2} / b^{2}+n^{2} / c^{2}\right) t} \sin \frac{m \pi x}{b} \sin \frac{n \pi y}{c}
$$

where

$$
a_{m n}=\int_{0}^{b} \int_{0}^{c} f\left(x^{\prime}, y^{\prime}\right) \sin \frac{m \pi x^{\prime}}{b} \sin \frac{n \pi y^{\prime}}{c} d x^{\prime} d y^{\prime}
$$

In order to prove that the double series (79) is really the desired solution, we must show that it converges and defines a continuous function of $x, y$, and $t$ in the region

$$
0 \overline{\bar{\Sigma}} x \overline{\overline{<}} b, \quad 0 \overline{\overline{<}} y \overline{\overline{<}} c, \quad t>0,
$$

that it satisfies throughout this region the equation

$$
\frac{\partial u}{\partial t}=a^{2}\left(\frac{\partial^{2} u}{d x^{2}}+\frac{\partial^{2} u}{\partial y^{2}}\right)
$$

that it approaches $f\left(x_{1}, y_{1}\right)$ as $t$ approaches zero through positive values and

* See Fejer, l. c., p. 60.

† Cf. BYERLY, An Elementary Treatise on Fourier's Series, etc., p. 142, ex. 4. An analogous problem in three dimensions that can be treated rigorously by means of the generalizations referred to in the Introduction, in a manner precisely similar to the present treatment, may be found in Carsusw, Fourier's Series and Integrals, p. 307. 
$x$ and $y$ approach $x_{1}$ and $y_{1}$, provided $\left(x_{1}, y_{1}\right)$ is a point in the interior of a region throughout which $f(x, y)$ is continuous, and that it remains finite when $t$ approaches zero through positive values and $x$ and $y$ approach the coördinates of any point of the region.

$$
0 \bar{\gtrless} x \overline{<} b, \quad 0 \overline{<} y \bar{\gtrless} c .
$$

That the series (79) is convergent, and in fact absolutely convergent in the region (81), provided $f(x, y)$ is finite and integrable in the region (83), is easily shown. For, on account of the presence of the convergence factor

$$
e^{-a^{2} \pi^{2}\left(m^{2} / b^{2}+n^{2} /(c) s\right)} \text {, }
$$

we have for the general term, $u_{m n}(x, y, t)$, of the series

$$
\left|u_{m n}(x, y, t)\right|<\frac{K}{m^{2} n^{2}} \quad\left(0 \leqq x \leqq b, 0 \leqq y \leqq c, 0<t_{0} \leqq t\right),
$$

where $K$ is a positive constant and $t_{0}$ a positive constant as small as we please, provided $f(x, y)$ is finite and integrable in (83). Since the right-hand side of (85) is the general term of a convergent double series of positive terms, it follows that (79) converges absolutely in the region

$$
0 \overline{\bar{k}} x \overline{\bar{k}} b, \quad 0 \overline{\bar{k}} y \overline{\bar{k}} c, \quad 0<t_{0} \overline{<} t,
$$

and therefore, since $t_{0}$ is arbitrary, in the region (81). That the function it defines there is continuous can also be shown by means of the inequality (85) for it follows from this inequality and the test for uniform convergence stated at the end of $\S 1$, that (79) is uniformly convergent in the region (86). Since also its terms are continuous there, it follows that it defines a continuous function in (86), or since $t_{0}$ is arbitrary, in (81).

We wish next to show that the function defined by (79) satisfies the equation (82) throughout the region (81). Each term of the series obviously satisfies this equation, and hence if we can show that we have a right to form the several derivatives involved by differentiating the series term by term, it will follow that the function defined by the series satisfies this equation. The proof that we have a right to differentiate the series term by term is similar to the proof of the continuity of the function defined by the series, the principal difference being that the present proof rests on the uniform convergence of the derived series and the theorem that we have a right to differentiate a convergent double series if the resulting series is uniformly convergent in the region under consideration. The details of this proof are easily carried through.

It remains finally to show that when $t$ approaches zero through positive 
values, and $x$ and $y$ approach $x_{1}$ and $y_{1}$, the function defined by the series will approach $f\left(x_{1}, y_{1}\right)$ when $\left(x_{1}, y_{1}\right)$ is an interior point of a region of continuity of $f(x, y)$, and will remain finite when this is not the case.

In order to establish these facts we must make use of some of the results we have obtained with regard to convergence factors in double series. It will be observed that the convergence factors of the form (84) are, for any particular values of $m$ and $n$, functions of a single variable $t$, whereas the convergence factors we have dealt with previously were functions of two variables.* However, there was nothing in the conditions of the theorems on convergence factors to prevent there being a relation between the two arguments of the convergence factors, and if we set

$$
\alpha=\frac{a^{2} \pi^{2} t}{b^{2}}, \quad \beta=\frac{a^{2} \pi^{2} t}{c^{2}},
$$

the convergence factors of the form (84) assume the form

$$
e^{-\left(m^{2} a+n * \beta\right)} \text {, }
$$

a form to which the conditions of Theorem II can be applied at once.

Our results on convergence factors must be used in connection with some of the results we have obtained with regard to the summability of the double Fourier's series, in order to establish the facts in question. It follows from Corollary 1 of Theorem IV by a change of variable, that the series (79) without the convergence factors is uniformly summable $(C 1)$ to $f(x, y)$ throughout some small region surrounding any interior point of a region of continuity of $f(x, y)$. Hence if we can show that the convergence factors of the form (88) satisfy the conditions of Theorem II, it will follow from the corollary of that theorem that the function defined by (79) approaches $f(x, y)$ uniformly throughout the small region above referred to, as the $\alpha$ and $\beta$ defined by (87) approach +0 , or what is the same thing, as $t$ approaches +0 . From this we shall be able to conclude at once that the function defined by (79) approaches $f\left(x_{1}, y_{1}\right)$ as $x$ and $y$ approach $x_{1}$ and $y_{1}$ and $t$ approaches +0 , provided $\left(x_{1}, y_{1}\right)$ is an interior point of a region of continuity of $f(x, y)$.

It only remains then, in order to establish the result just stated, to show that the convergence factors of the form (88) satisfy the conditions of Theorem II. These conditions include the conditions of Lemma 3 and the conditions $(e)$ and $(f)$. It is obvious that conditions $(e)$ and $(f)$ and condition $(d)$ of Lemma 3 are satisfied; it remains to be shown that conditions $(a),(b)$, and (c) of Lemma 3 are satisfied.

* Our object in considering convergence factors that were functions of two variables was, of course, to obtain results of greater generality. 
Let us consider first condition (b). We have obviously

$$
\left|e^{-\left(m^{2} a_{0}+n^{2} \beta_{0}\right)}\right|<\frac{K}{m^{4} n^{4}} \quad(m, n=1,2,3, \cdots),
$$

where $K$ is a positive constant, provided $\alpha_{0}$ and $\beta_{0}$ are any constants greater than zero. Hence

$$
\sum_{j=\mu}^{j=\nu} j\left|e^{-\left(m^{2} a_{0}+j^{2} \beta_{0}\right)}\right|<\sum_{j=\mu}^{j=\nu} \frac{K j}{m^{4} j^{4}}<\frac{K}{m^{4}} \sum_{j=1}^{\infty} \frac{1}{j^{3}}=\frac{K_{1}}{m^{4}},
$$

where $K_{1}$ is a positive constant, and hence condition (b) of Lemma 3 is satisfied by the convergence factors of the form (88). That condition (c) is satisfied may be shown in a manner entirely similar, and hence it only remains to be proved that condition $(a)$ is satisfied.

We have by direct computation

$$
\Delta_{2}^{2} e^{-\left(m^{2} a+n^{2} \beta\right)}=\left[e^{-m^{2} \alpha}-2 e^{-(m+1)^{2} \alpha}+e^{-(m+2)^{2} a}\right] \cdot\left[e^{-n^{2} \beta}-2 e^{-(n+1)^{2} \beta}+e^{-(n+2)^{2} \beta}\right] .
$$

It follows then that

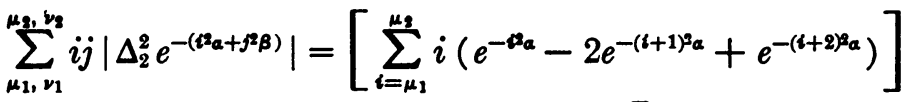

$$
\begin{aligned}
& \cdot\left[\sum_{j=\nu_{1}}^{\nu_{8}} j\left(e^{-j 2 \beta}-2 e^{-(j+1)^{\alpha} \beta}+e^{-(j+2)^{2} \beta}\right)\right] \text {, }
\end{aligned}
$$

and hence (a) will hold for some $K$ provided $K$ can be so chosen that

$$
\sum_{i=1}^{\infty} i\left|e^{-i u_{u}}-2 e^{-(i+1)^{2} u}+e^{-(i+2)^{2} u}\right|<\sqrt{K} \quad(u>0) .
$$

Therefore it only remains to be shown that the inequality (90) holds for a proper choice of $K$.

We have evidently

$$
e^{-i t^{2}}-2 e^{-(i+1)^{2} u}+e^{-(i+2)^{2} c}=e^{-(i+\theta)^{2} u}\left\{4(i+\theta)^{2} u^{2}-2 u\right\} \quad(0<\theta<2),
$$

whence it follows that the terms in the series

$$
\sum_{i=1}^{\infty} i\left(e^{-i n}-2 e^{-(i+1)^{2} u}+e^{-(i+2)^{2} u}\right)
$$

are negative for $i=1,2, \cdots$, so long as $(i+2)$ is less than $(2 u)^{-1 / 2}$, and are positive for all values of $i$ greater than $(2 u)^{-1 / 2}$. Thus the series consists of a group of negative terms followed by two terms of uncertain sign and an infinite set of positive terms. But it follows at once from (91), that a positive constant can be assigned such that each individual term of the series is less in absolute value than this constant. Hence $K$ can be chosen so that the 
inequality (90) holds, provided any sequence of positive or of negative terms that we can choose from (92) is less in absolute value than a fixed, positive constant. Any such set of terms

$$
p\left(e^{-p^{2} u}-2 e^{-(p+1)^{2} u}+e^{-(p+2)^{2} u}\right)+\cdots+q\left(e^{-q^{2} u}-2 e^{-(q+1)^{2} u}+e^{-(q+2)^{2} u}\right)
$$

has the sum

$$
p e^{-p^{2} u}-(p-1) e^{-(p+1)^{2} u}-(q+1) e^{-(q+1)^{2} u}+q e^{-(q+2)^{2} u}
$$

which differs from

$$
p\left(e^{-p^{2} u}-e^{-(p+1)^{2} u}\right)-(q+1)\left(e^{-(q+1)^{2} u}-e^{-(q+2)^{2} u}\right)
$$

by at most unity. But

$$
\begin{aligned}
\left|m\left(e^{-m m^{2} u}-e^{-(m+1)^{0} u}\right)\right|=\mid m e^{-(m+\theta)^{2} u} & \{-2(m+\theta) u\} \mid \\
& <2(m+\theta)^{2} u e^{-(m+\theta)^{2} u} \quad(0<\theta<1),
\end{aligned}
$$

and since $\rho e^{-\rho}$ is limited for $\rho>0$, the left-hand member of (95) is limited for all positive, integral values of $m$ and all values of $u>0$. Hence the same is true of (94) and (93), and therefore any sequence of positive or negative terms chosen from (92) is less in absolute value than a fixed positive constant for all values of $u>0$. Hence (90) holds, and consequently, as we have pointed out before, condition $(a)$ is satisfied.

We have now shown that the convergence factors of the form (88) satisfy all the conditions of Theorem II. It therefore follows, as we have pointed out before, that the function defined by (79) approaches $f\left(x_{1}, y_{1}\right)$ as $x$ and $y$ approach $x_{1}$ and $y_{1}$ and $t$ approaches +0 , provided $\left(x_{1}, y_{1}\right)$ is an interior point of a region of continuity of $f(x, y)$. It remains to be shown that the function defined by (79) remains finite as $t$ approaches +0 and $x$ and $y$ approach the coördinates of any point in the region (83) in order to complete the proof that this function furnishes the desired solution of our physical problem.

We have seen that the convergence factors of the form (88) satisfy all the conditions of Lemma 3. It follows then from that lemma that for all positive values of $\alpha$ and $\beta$, that is to say for all values of $t>0$, the series

$$
\sum_{i=1, j=1}^{\infty, \infty} S_{i j}^{(1)}(x, y) \Delta_{2}^{2} e^{-\left(i \mathbf{a} a+j^{2} \beta\right)}
$$

converges to the same value as the series (79). But, since $f(x, y)$ is finite and integrable in the region (83), it follows from Theorem $\mathrm{V}$ that

$$
\left|S_{i j}^{(1)}(x, y)\right|<C i j \quad\left(\begin{array}{l}
i, j=1,2,3, \cdots \\
0 \leqq x \leqq b, 0 \leqq y \leqq c
\end{array}\right)
$$


where $C$ is a positive constant. Combining this result with the fact that condition (a) of Lemma 3 is satisfied by the convergence factors in (79), we have

$$
\begin{aligned}
& \left|\sum_{i=1, y=1}^{\infty, \infty} S_{i j}^{(1)}(x, y) \Delta_{2}^{2} e^{-\left(p_{a}+j^{\beta} \beta\right)}\right| \sum \sum_{i=1, j=1}^{\infty}\left|S_{i j}^{(1)}(x, y)\right| \cdot\left|\Delta_{2}^{2} e^{-\left(p_{a}+j^{\beta} \beta\right)}\right|
\end{aligned}
$$

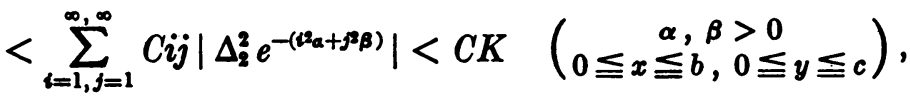

and hence it follows that the series (96) remains finite for all positive values of $\alpha$ and $\beta$ and all values of $x$ and $y$ in the region (83). But since the series (79) converges to the same value as the series (96) for all values of $t>0$, it follows that the function defined by the series (79) remains finite as $t$ approaches +0 and $x$ and $y$ approach the coördinates of any point in the region (83). Hence the function defined by (79) furnishes the desired solution of our physical problem.

Paris,

December, 1911. 